\title{
Partial replacement of fishmeal with Spirulina platensis and Chlorella vulgaris and its effect on growth and body composition of African catfish Clarias gariepinus (Burchell 1822)
}

\author{
AMEENAT ABIODUN RAJI ${ }^{1,4}$, OLAWALE QUAZIM JUNAID², POZI MILOW ${ }^{1}$, NORHIDAYAH \\ MOHD. TAUFEK ${ }^{1}$, ADAMU MISAU FADA ${ }^{3}$, ASIATA ADEKILEKUN KOLAWOLE ${ }^{2}$, ZAZALI $^{2}$ \\ ALIAS ${ }^{1}$ AND SHAHARUDIN ABDUL RAZAK ${ }^{1}$ \\ ${ }^{1}$ Institute of Biological Sciences, Faculty of Sciences, University of Malaya, 50603, Kuala Lumpur, Malaysia \\ ${ }^{2}$ Department of Biological Sciences, Faculty of Science, Federal University Kashere, Gombe State, Nigeria \\ ${ }^{3}$ Fisheries Department, Bauchi State Agricultural Development Programme, Bauchi State, Nigeria \\ ${ }^{4}$ Science Laboratory Technology Department, School of Applied Sciences, Federal Polytechnic Ede, Osun State, Nigeria \\ e-mail: ameenatabiodunraji.um19@gmail.com
}

\begin{abstract}
The study examined the effect of partial replacement of fishmeal with Spirulina platensis (SP) and Chlorella vulgaris (CL) powder in the diets of African catfish Clarias gariepinus (Burchell 1822) fingerlings on growth performance and body composition. Nine diets (45.14-48.45\% crude protein) consisting SP or CL as fishmeal replacement at zero (control), 12.5, 25,50 and $75 \%$, were fed to triplicate groups of 10 fingerlings $(7.82 \pm 0.08 \mathrm{~g})$ each for 56 days. The results showed statistically higher weight gain, specific growth rate, protein efficiency ratio, relative growth rate, Fulton's condition factor $(\mathrm{K})$ and a corresponding lower feed conversion ratio when the replacement of C. vulgaris was at $75 \%$. Proximate analysis revealed a significantly greater carcass protein in the SP12.5\% and the highest flesh lipid and gross energy in SP75\% groups. Flesh lipid increased with increasing SP and CL levels. Polynomial curve estimation regression analysis revealed the optimum fishmeal replacement levels for best growth are at 68.5 and $69.4 \%$ for SP and CL, respectively. CL was found to be more efficient in terms of growth and effective feed utilisation than SP.
\end{abstract}

Keywords: Body composition, Chlorella vulgaris, Fishmeal, Growth, Spirulina platensis

\section{Introduction}

Increase in human population and overexploitation of natural water bodies have reduced the levels of fish captured from wild and led to increase in aquaculture production. Aquaculture is among the fastest growing food producing sectors globally which forms a potential solution to the reduction in global capture fisheries production (Lehane, 2013). The cost of feed in aquaculture production has led to reliance on feed ingredients, especially fishmeal (FM), due to its high profile of proteins and growth factors (NRC, 2011). The increased demand for fishmeal together with its static production necessitated the search for alternative, locally available protein sources as its replacement in fish feed.

Previously, researchers used plant protein as a replacement for the conventional fishmeal diet in feeding fish. These include various pulses and lupins in various fishes. However, soybean meal and related byproducts have been widely used in fish diet and are of prime importance in the tropical omnivorous fish feed (Goda et al., 2007; Jimoh et al., 2014). However, microalgae are mainly used in aquaculture nutrition as a sole component or food additive, for colouring the flesh of salmonids and for inducing other biological activities (Richmond, 2004). Microalgae have high protein content and digestible amino acid profiles comparable with those of other reference food proteins (Becker, 2007; Reyes-Becerril et al., 2013). They are also high in polyunsaturated fatty acids, $\beta$-carotene, antioxidants, sulphated polysaccharides and sterols (Otles and Pire, 2001; Xue et al., 2002). Numerous studies have been conducted on the usage of algae on different cultured fish as additives. Many of these studies demonstrated that algae as feed additives improved the growth and feed utilisation of the cultured fish because of effective absorption of dietary protein, enhancement of biological activities, stress reduction and disease resistance (Gatlin, 2007; Guroy et al., 2011). Chlorella and Spirulina have great potential to be used in aquafeed due to their high protein content, indispensable amino and fatty acids, antioxidant pigments as well as immunostimulatory properties (Becker, 2007; Lordan et al., 2011).

Chlorella vulgaris is a unicellular green alga that is very rich in chlorophyll, protein $(51-58 \%$, depending on 
the species), crude lipid (14-15\%) and essential nutrients like polyunsaturated fatty acid pigments, antioxidant vitamins such as B6, B12, $\beta$-carotene $\mathrm{C}, \mathrm{E}$ and minerals (Tartiel, 2005; Becker, 2007; Badwy et al., 2008). More importantly, amino acids such as aspartic acid, serine, alanine, leucine and glycine were found to contribute to $50 \%$ of dry matter of Chlorella (Badwy et al., 2008). It also contains Chlorella growth factor (CGF) and $\beta-1$, 3-glucan, a strong immune stimulator, a free-radical scavenger as well as a reducer of blood fat (Spolaore et al., 2006). Previously, researchers have investigated Chlorella as a feed additive or fishmeal replacement for growth enhancement and as in immune booster of different fish species such as Gibel carp (Carassius auratus gibelio) (Qihuan et al., 2014; Xu et al., 2014), rohu (Labeo rohita) (Pradhan and Das, 2015), Chlorella and Scenedesmus on Nile tilapia (Oreochromis niloticus) (Badwy et al., 2008).

On the other hand, Spirulina platensis is a bluegreen cyanobacterium that is very rich in protein, $55-70 \%$ depending on the species and the source (Phang et al., 2000; Lordan et al., 2011), essential fatty acids (GLA) and vitamins, essentially pro-vitamin A and B12 (Belay, 2002; Gouveia et al., 2008; Dalle Zotte et al., 2013). It is also rich in antioxidant pigments, especially phycocyanin and chlorophyll (Wang et al., 2007). Previous researchers have examined the influence of Spirulina on the development, immune responses and nutrient utilisation of different fish species such as Clarias gariepinus, Oplegnathus fasciatus and Cyprinus carpio (Promya and Chitmanat, 2011; Abdulrahman and Ameen, 2013; Kim et al., 2013; Abdulrahman and Ameen, 2014; Sayed and Fawzy, 2014).

African catfish Clarias gariepinus (Burchell 1822) is regarded as one of the most important freshwater fish due to its fast growth, reproduction and adaptability in Africa, South-east Asia and other parts of the world (Fasakin et al., 2003; Al-Dohail et al., 2009). It has superior growth performance compared with other Clarias species. Hence, there is a need for a well-balanced diet to sustain and increase the commercial production of this important fish species at a reasonable cost to both farmers and consumers. Information available in literature regarding optimum dietary addition levels of C. vulgaris and $S$. platensis for the growth performance of most fish species are inconsistent. There is also scarce information on the effects of $C$. vulgaris on $C$. gariepinus. This may be partly attributed to the fact that $C$. vulgaris and $S$. platensis diets are yet to be commercially applied as a source of macronutrients in fish feed formulation (Teuling et al., 2017). The high nutritional and health properties of the two algae and their usefulness to growth and body composition of fish has attracted much research attention. But, no published information is available on the effect of C. vulgaris or S. platensis in African catfish. Thus, this study included $C$. vulgaris and S. platensis separately in pelleted diets, to ascertain the effects of their altered inclusion levels on growth enhancement and carcass composition of $C$. gariepinus. Additionally, the study sought to determine the comparative advantages of one alga over the other, as well to establish the optimal level that could positively replace fishmeal without adverse effect on C. gariepinus growth.

\section{Materials and methods}

\section{Experimental diets}

Spray-dried and thin cell C. vulgaris (cell-ruptured) and $S$. platensis powder used for this study were purchased from TST Bioceuticals (Perak, Malaysia). The powder was used to formulate nine experimental diets based on the protein requirement of clariid fish fingerlings. The diets were prepared in sets of either Chlorella (CL) or Spirulina (SP) to replace fishmeal at various inclusion levels of $0,12.5,25,50$ and $75 \%$ resulting in diet levels of SP $12.5 \%$ to SP $75 \%$ or CL12.5\% to CL $75 \%$. Fishmeal was replaced proportionally according to their inclusion level, while other feed ingredients were adjusted to attain isonitrogenous and caloric diets (Table 1). Diets were formulated using Pearson's square and Winfeed 2.8 software (Mirza, 2004). After milling essential feed components with a hammer mill (Disk Mill, FFC 454), vitamins, minerals and di-calcium phosphate (DCP) were carefully mixed using water, then pelleted into $1 \mathrm{~mm}$ diameter in a pelleting machine (model KCM, Y132M-4). The pelletised feed was oven dried at $70^{\circ} \mathrm{C}$ and preserved in a feed cold room $\left(4^{\circ} \mathrm{C}\right)$ until use.

\section{Experimental set-up}

A total of 450 African catfish fingerlings were purchased from a native supplier in Balakong Hatchery, and acclimatised at the Institute of Biological Sciences Freshwater Aquarium Laboratory, University Malaya. Prior to stocking, the fingerlings $(7.82 \pm 0.08 \mathrm{~g})$ were starved for $24 \mathrm{~h}$ and 270 fingerlings were randomly stocked into 27 different tanks, with 10 fish per tank and 3 replicates per treatment. Experimental fish were fed twice daily between 9:00 and 16:00 hrs at 4\% body weight for 56 days. Fish sampling and weighing were performed fortnightly. The tanks were monitored closely for mortality and dead fishes were removed and recorded for determination of survival rate. On the commencement of the feeding trials, 10 fish samples were homogenised, 
Table 1. Ingredients and proximate composition ( $\%$ as fed) of experimental diets

\begin{tabular}{|c|c|c|c|c|c|c|c|c|c|}
\hline \multirow{3}{*}{ Ingredients $\left(\mathrm{g} \mathrm{kg}^{-1}\right)$} & \multicolumn{9}{|c|}{ Treatments (Replacement \%) } \\
\hline & \multirow{2}{*}{$\begin{array}{l}\text { Control } \\
0 \%\end{array}$} & \multicolumn{4}{|c|}{${ }^{*}$ Spirulina platensis } & \multicolumn{4}{|c|}{${ }^{* *}$ Chlorella vulgaris } \\
\hline & & $12.5 \%$ & $25 \%$ & $50 \%$ & $75 \%$ & $12.5 \%$ & $25 \%$ & $50 \%$ & $75 \%$ \\
\hline Fishmeal & 250 & 218.8 & 187.5 & 125 & 62.5 & 218.8 & 187.5 & 125 & 62.5 \\
\hline Spirulina & 0 & 31.2 & 62.5 & 125 & 187.5 & 0 & 0 & 0 & 0 \\
\hline Chlorella & 0 & 0 & 0 & 0 & 0 & 31.2 & 62.5 & 125 & 187.5 \\
\hline Soya bean & 503 & 494.5 & 501.9 & 500.3 & 501.9 & 512.3 & 521.7 & 540.5 & 559.2 \\
\hline Rice bran & 139.3 & 150.9 & 143.9 & 148.5 & 143.9 & 133.6 & 127.9 & 116.5 & 105.1 \\
\hline Premix $^{a}$ & 9 & 9 & 9 & 9 & 9 & 9 & 9 & 9 & 9 \\
\hline Cod liver oil & 59.7 & 56.6 & 56.2 & 53.2 & 56.2 & 56.1 & 52.4 & 45.1 & 37.7 \\
\hline Supplements+binder ${ }^{\mathrm{b}}$ & 39 & 39 & 39 & 39 & 39 & 39 & 39 & 39 & 39 \\
\hline Total & 1000 & 1000 & 1000 & 1000 & 1000 & 1000 & 1000 & 1000 & 1000 \\
\hline \multicolumn{10}{|c|}{ Nutrient levels (\% Dry matter basis) } \\
\hline Protein & 47.84 & 48.45 & 46.74 & 45.58 & 45.59 & 45.14 & 45.53 & 46.35 & 46.03 \\
\hline Fat & 9.27 & 10.62 & 9.91 & 8.87 & 8.92 & 10.48 & 8.43 & 8.07 & 7.37 \\
\hline Ash & 8.87 & 9.28 & 8.49 & 8.09 & 8.27 & 8.98 & 8.53 & 8.00 & 7.30 \\
\hline Moisture & 6.49 & 4.29 & 5.94 & 5.98 & 8.27 & 6.51 & 6.69 & 6.19 & 6.70 \\
\hline Crude fibre & 2.02 & 3.19 & 1.76 & 2.29 & 1.29 & 2.89 & 1.99 & 2.37 & 2.12 \\
\hline NFE & 25.51 & 22.17 & 27.16 & 29.19 & 27.66 & 26.00 & 30.83 & 29.02 & 30.48 \\
\hline Gross energy $\left(\mathrm{KJ} \mathrm{g}^{-1}\right)$ & 19.24 & 19.53 & 19.51 & 19.17 & 18.94 & 19.17 & 18.98 & 19.05 & 18.99 \\
\hline
\end{tabular}

${ }^{\text {a }}$ Vitamin and Mineral premix supplied: vitamins A - 500IU; B1 - $1.0 \mathrm{mg}$; B2 - $0.5 \mathrm{mg}$; B3 - $0.3 \mathrm{mg}$; B6 - $0.2 \mathrm{mg}$; B12 - $0.001 \mathrm{mg}$; C - $0.1 \mathrm{mg}$. D3 - 100 IU; E - 0.75 mg, K - 0.02 mg; Niacin - 0.2 mg, Folic acid - $0.1 \mathrm{mg}$; Biotin - 0.24 mg; Pantothenic acid - $1.0 \mathrm{mg}$; Inositol - $2.5 \mathrm{mg}$; Iron - 8.0 mg; Selenium - 0.2 mg; Magnesium oxide - 0.6 mg; Manganese - $1.0 \mathrm{mg}$; Zinc -8.0 mg; Copper - $0.15 \mathrm{mg}$; Potassium chloride - $0.4 \mathrm{mg}$; Sodium bicarbonate - $1.5 \mathrm{mg}$; Iodine - $1.0 \mathrm{mg}$; Cobalt - $.25 \mathrm{mg}$

${ }^{\mathrm{b}}$ Supplements plus binder: Lysine, Methionine, DCP and binder at $0.2 ; 0.7 ; 1.0 ; 20$, respectively

*Spirulina proximate composition: CP 60.71\%; Crude lipid 8.68\%; Energy 477.92 Kcal $100 \mathrm{~g}^{-1}$

${ }^{* *}$ Chlorella proximate composition: CP 58.0\%; Crude lipid 9.59\%; Energy $478.05 \mathrm{Kcal} 100 \mathrm{~g}^{-1}$

Fishmeal proximate composition: CP $66.67 \%$; Crude lipid 9.55\%; Energy $472.21 \mathrm{Kcal} 100 \mathrm{~g}^{-1}$

then frozen while 3 samples per replicate were euthanised with $10 \%$ clove oil, for subsequent carcass examination on termination of the experiments.

\section{Proximate examination of trial diets and fish carcass}

Proximate analysis (crude protein, dry matter, ash, crude fibre and crude lipid) was carried out on the ingredients, formulated diets and fish carcass as per AOAC (2000). Crude protein analysis was done via the Kjeldahl method $(\mathrm{N} \times 6.25)$ after acid digestion using Vapodest 50 (Gerhardt Germany). Dry matter and moisture were determined through oven drying at $105^{\circ} \mathrm{C}$ to a constant weight and ash via combustion in a muffle furnace (Memmert UFB500 and Carbolite Furnace Memmert CWF 11/13, Germany) at $600^{\circ} \mathrm{C}$. Crude lipid was determined as per Bligh and Dyer (1959) with slight modification. This involved dissolving the sample in a mixture of 2:1 chloroform and methanol in Soxhlet apparatus (Gerhardt Soxtherm) for $8 \mathrm{~h}$ for SP and CL and petroleum ether extraction for other samples for $1.5 \mathrm{~h}$. Fiber contents were determined by alkali and acid digestion of lipid residue. Nitrogen free extract (NFE) was calculated as $100-(\%$ crude lipid $+\%$ crude fibre $+\%$ crude protein
$+\%$ crude ash) and gross energy $\left(\mathrm{kJ} \mathrm{g}^{-1}\right)$ was calculated using the physiological values, CP x $23.9+$ lipid $\times 39.8+$ carbohydrates $\times 17.6$ (Schulz et al., 2005). The essential amino acid composition of freeze-dried algae meal was determined by high-performance liquid chromatography (HPLC) equipped with a fluorescence detector (Taufek et al., 2016) and the contents were quantified using the Pico-tag technique by Heinrikson and Meredith (1984). Meanwhile, the alkaline hydrolysis method by Nielsen and Hurrell (1985) was used to determine tryptophan. Fatty acid methyl esters were separated and measured following the method by Ichihara and Fukubayashi (2010) using Agilent 7820A gas chromatograph fitted with a capillary column (SLB-IL 100, $30 \mathrm{~m} \times 0.25 \mathrm{~mm} \times$ $0.20 \mu \mathrm{m}$, Supelco, USA) plus a flame ionisation detector (FID) using the temperatures of injector and detector at 250 and $260^{\circ} \mathrm{C}$, respectively. The different FAMEs were recognised by comparing their retention times with those of known standards from Sigma-Aldrich, USA, while fatty acids were quantified in milligram per gram of lipids, by addition of the internal standard C7:0 Sigma, USA (Vello et al., 2014). 
Mean weight gain $=\frac{\mathrm{Wf}-\mathrm{Wi}}{\mathrm{n}}$

where, $\mathrm{Wf}=$ final weight; $\mathrm{Wi}=$ initial weight and $\mathrm{n}=$ number of fish.

Relative growth rate $(\mathrm{RGR})=\frac{\mathrm{Wg}}{\mathrm{Wi}} \times 100$

where, $\mathrm{Wg}=$ weight gain and $\mathrm{Wi}=$ initial weight

Specific growth rate $(\mathrm{FCR})=\frac{(\log \mathrm{Wf}-\log \mathrm{Wi})}{\mathrm{t}} \times 100$

where, $\log \mathrm{Wf}=\log$ of final weight, $\log \mathrm{Wi}=\log$ of initial weight, and $\mathrm{t}=$ time .

Feed conversion ratio $(\mathrm{FCR})=\frac{\mathrm{Fi}}{\mathrm{FWg}}$

where, $\mathrm{Fi}=$ dry feed fed and $\mathrm{FWg}=$ fish wet weight gain.

Protein efficiency ratio $(\mathrm{PER})=\frac{\mathrm{MWG}}{\mathrm{MPI}}$

where, $\mathrm{MWG}=$ mean weight gain and MPI = mean protein fed.

Survival rate $=\frac{F n}{I n} \times 100$

where, $F n$ = final quantity of fish at the end of experiment and In $=$ initial number of fish at the beginning of experiment.

Protein productive value $(\mathrm{PPV})=\frac{\mathrm{FPE}}{\mathrm{FPB}} \times 100$

where, $\mathrm{FPE}=$ total fish protein at the end and $\mathrm{FPE}=$ total fish protein at the beginning of feeding experiment.

Condition $(\mathrm{K})$ factor $=\frac{\mathrm{FW}}{\mathrm{L}^{3}} \times 100$

where, $\mathrm{W}=$ the weight of fish $(\mathrm{g})$ and $\mathrm{L}=$ standard length $(\mathrm{cm})$ (Htun-Han, 1978).

\section{Growth performance indices}

Fish weights and feed supplied were measured and recorded fortnightly to compute the growth indices following the method by Oliva-Teles and Goncalves (2001).

\section{Statistical analysis}

One-way analysis of variance (ANOVA) with SPSS version 21 statistical package (SPSS Inc., Chicago) was used to analyse the experimental data. Duncan's multiple range analysis (Duncan, 1955) was used to compare differences between treatments and considered to be significant when $p<0.05$. Curve estimation (polynomial) regression analysis was performed with the aid of Wolfram Mathematical 11 version (11:0.1.0) student edition (Wolfram Research Inc., Champaign IL, USA), to estimate the optimum level of dietary replacement with
CL and SP respectively relative to the weight gain of C. gariepinus fingerlings.

\section{Results}

Proximate composition of Spirulina platensis, Chlorella vulgaris and experimental diets

Table 1 shows the formulations and proximate composition of the different diets used in the study. The crude protein of the experimental diets $(\%)$ ranged from 45.14 to 48.45 , lipid 7.37 to 10.62 , ash 7.30 to 9.28 , moisture 4.29 to 8.27 , fiber 1.29 to 3.19 , NFE 22.18 to 30.84 and gross energy 18.94 to $19.53 \mathrm{KJ} \mathrm{g}^{-1}$. This can be compared to the composition of S. platensis (CP 60.71\%; crude lipid 8.68\%; energy $20.00 \mathrm{KJ} \mathrm{g}^{-1}$ ), C. vulgaris (CP 58.0\%; crude lipid 9.59\%; energy $20.00 \mathrm{KJ} \mathrm{g}^{-1}$ ) and fishmeal (CP 66.67\%; crude lipid 9.55\%; energy $19.76 \mathrm{KJ} \mathrm{g}^{-1}$ ) used for the diets (Table 1).

Significant differences $(\mathrm{p}<0.05)$ were observed in total fatty acids, saturated fatty acids, total monounsaturated fatty acids, total n-6 and n-3 (18, PUFAs), n-3 and n-6 long chain (LC) PUFAs among dietary treatments (Table 2). The CL12.5\% diet had significantly $(p<0.05)$ higher total fatty acids (TFA) than control and other treatments, while CL50\% diet had significant higher $(p<0.05)$ linoleic acid, C18:2n6 $(20.11 \%)$. Saturated fatty acids (SFA), the majority of which was made up of palmitic acid $(\mathrm{C} 16: 0)$ was significantly higher $(\mathrm{p}<0.05)$ in the SP50\% diet, whereas of the total monounsaturated fatty acids that predominated in SP12.5\% (24.33\%), a substantial amount was made up of oleic acid (18:1n9c) as found in SP25\% (12.61\%). Both linolenic and docosahexaenoic (18:3n3 and 22:6n3DPA) acids were higher in CL25\%, while eicosapentaenoic acid (EPA), 20:5n3 was higher in the CL75\% diet.

All the 10 essential amino acids were present in varying amounts in all the diets (Table 3 ). Among the diets, SP50\% was considerably greater $(\mathrm{p}<0.05)$ in histidine, arginine, threonine, valine, methionine, isoleucine, leucine and phenylalanine. Lysine and tryptophan were significantly $(\mathrm{p}<0.05)$ higher in CL12.5\% diet.

\section{Growth performance}

The growth performance of CL75\% fish were significantly $(p<0.05)$ higher in terms of body weight gain $(16.32 \pm 0.01 \mathrm{~g})$, SGR $(3.15 \pm 0.00 \%)$, PER (2.33 \pm 0.01$)$, RGR (209.02 $\pm 1.05 \%), \mathrm{K}$ factor $(1.89 \pm 0.02)$ and a corresponding lower FCR $(0.93 \pm 0.01)$, while SP12.5\% recorded highest $(p<0.05)$ PPV of $83.61 \pm 0.04$ (Table 4). However, optimum level of replacement of fishmeal with CL relative to the growth of the fish was found to be $69.4 \%$, while optimum replacement with SP was $68.5 \%$ (Fig. 1a and b). 
Table 2. Fatty acid composition ( $\left.\mathrm{mg} \mathrm{g}^{-1}\right)$ of diets containing various levels of S. platensis and C. vulgaris

\begin{tabular}{|c|c|c|c|c|c|c|c|c|c|}
\hline Fatty & Control & $.5 \%$ & $25 \%$ & P50\% & $\%$ & $\%$ & CL25\% & CL50\% & CL75\% \\
\hline$\sum F A S$ & $.62 \pm 0.01$ & $.47 \pm 0.01$ & $8.81 \pm 0.02$ & $.31 \pm 0.01$ & $8.46 \pm 0.02$ & $2.48 \pm 0.01$ & $.70 \pm 0.01$ & $13.39 \pm 0.00$ & .01 \\
\hline${ }^{1} \sum \mathrm{SFA}$ & $46.08 \pm 0.01^{\mathbf{b}}$ & $7.59 \pm 0.02^{\mathrm{c}}$ & $47.83 \pm 0.01^{\mathrm{d}}$ & $54.28 \pm 0.01^{\mathrm{i}}$ & $1.15 \pm 0.02^{\mathrm{h}}$ & $47.91 \pm 0.01^{\mathrm{e}}$ & $40.49 \pm 0.01^{\mathrm{a}}$ & $50.11 \pm 0.00^{\mathrm{g}}$ & $48.38 \pm 0.01^{\mathrm{f}}$ \\
\hline${ }^{2} \sum \mathrm{M}$ & $20.00-0.04$ & $4.33 \pm 0.01^{\mathrm{i}}$ & $23.16 \pm 0.02^{h}$ & 80.02 & $20.12 \pm 0.02^{\mathrm{d}}$ & $22.47 \pm 0.0^{\mathrm{f}}$ & $46 \pm 0.01^{\mathrm{a}}$ & $19.71 \pm 0.01^{\mathrm{c}}$ & 18. \\
\hline${ }^{3} \sum$ PUFA & $30.89 \pm 0.02^{\mathrm{g}}$ & $8.12 \pm 0.21^{b}$ & & $24.73 \pm 0.02^{\mathrm{a}}$ & & $29.64 \pm 0.01^{\mathrm{e}}$ & & $30.17 \pm 0.01^{\mathrm{f}}$ & \\
\hline $\mathrm{C} 16.0$ & $27.90 \pm 0.01^{\mathbf{b}}$ & $\pm 0.02^{\mathrm{g}}$ & $29.75 \pm 0.02^{\mathrm{d}}$ & $35.90 \pm 0.02^{\mathrm{i}}$ & $2 \pm 0.01^{\mathrm{f}}$ & $=0.01^{\mathrm{e}}$ & $22.72 \pm 0.01^{a}$ & $32.43 \pm 0.02^{\mathrm{h}}$ & $\pm 0.02^{\mathrm{c}}$ \\
\hline C18.0 & $.01^{h}$ & $01^{\mathrm{c}}$ & $1^{d}$ & $1^{\mathrm{g}}$ & $01^{i}$ & $01^{\mathrm{f}}$ & $1^{\mathbf{b}}$ & $1^{\mathrm{e}}$ & $02^{\mathrm{a}}$ \\
\hline C18. & $=0.02^{\mathrm{d}}$ & $1^{\mathrm{g}}$ & $\pm 0.01^{\mathrm{i}}$ & $.01^{\mathrm{c}}$ & & $12.57 \pm 0.01^{\mathbf{h}}$ & & $02^{\mathrm{e}}$ & $0.02^{\mathrm{a}}$ \\
\hline${ }^{4} \sum \ln 9$ & $2^{g}$ & $\pm 0.01^{\mathrm{i}}$ & $19.75 \pm 0.01^{\mathrm{h}}$ & $16.99 \pm 0.01^{\mathrm{e}}$ & $\pm 0.01^{\mathrm{d}}$ & $18.84 \pm 0.01^{\mathbf{f}}$ & $12.48 \pm($ & $01^{\mathrm{c}}$ & $11.62 \pm 0.02^{\mathrm{a}}$ \\
\hline $\mathrm{C} 18.2 \mathrm{n} 6$ & $13.22 \pm 0.02^{\mathrm{d}}$ & $2.51 \pm 0.02^{\mathrm{c}}$ & $13.80 \pm 0.02^{\mathrm{e}}$ & $14.00 \pm 0.02^{\mathrm{f}}$ & $15.24 \pm 0.01^{\mathrm{h}}$ & $14.75 \pm 0.01^{\mathrm{g}}$ & $9.63 \pm 0.01^{\mathbf{b}}$ & $20.11 \pm 0.01^{\mathrm{i}}$ & $5.85 \pm 0.01^{\mathrm{a}}$ \\
\hline${ }^{5} \sum \mathrm{n}-6 \mathrm{PUFA}$ & $13.45 \pm 0.01^{\mathrm{d}}$ & $12.61 \pm 0.01^{\mathrm{c}}$ & $13.95 \pm 0.01^{\mathrm{e}}$ & $14.09 \pm 0.01^{\mathrm{f}}$ & $15.50 \pm 0.01^{\mathrm{h}}$ & $14.94 \pm 0.00^{\mathrm{g}}$ & $10.35 \pm 0.00^{\mathrm{b}}$ & $20.27 \pm 0.01^{\mathrm{i}}$ & $6.40 \pm 0.01^{\mathrm{a}}$ \\
\hline${ }^{6} \sum \mathrm{n}-3$ PUFA & $14.85 \pm 0.01^{\mathrm{g}}$ & $13.60 \pm 0.01^{\mathrm{f}}$ & $12.90 \pm 0.01^{\mathrm{e}}$ & $8.24 \pm 0.01^{\mathbf{b}}$ & 8. & $12.33 \pm 0.00^{d}$ & $32.61 \pm 0$ & $7.95 \pm 0.01^{\mathrm{a}}$ & $18.98 \pm 0.01^{h}$ \\
\hline $\mathrm{C} 20.5 \mathrm{n} 3$ & $5.93 \pm 0.01^{\mathrm{h}}$ & $5.69 \pm 0.01 \mathrm{~g}$ & $5.27 \pm 0.00^{\mathrm{f}}$ & $3.60 \pm 0.01^{\mathrm{c}}$ & $3.32 \pm 0.00^{\mathrm{a}}$ & $5.24 \pm 0.00^{\mathrm{e}}$ & $3.40 \pm 0.00^{\mathbf{b}}$ & $4.35 \pm 0.00^{d}$ & $7.84 \pm 0.00^{\mathrm{i}}$ \\
\hline $\mathrm{C} 22.6 \mathrm{n} 3$ & $5.46 \pm 0.00^{\mathrm{g}}$ & $5.21 \pm 0.00^{\mathrm{f}}$ & $4.72 \pm 0.00^{\mathrm{e}}$ & $2.74 \pm 0.01^{\mathrm{b}}$ & $2.39 \pm 0.01^{\mathrm{a}}$ & $4.23 \pm 0.01^{\mathrm{d}}$ & $27.72 \pm 0.00^{\mathrm{I}}$ & $6.00 \pm 0.01^{\mathrm{h}}$ & $3.75 \pm 0.01^{\mathrm{c}}$ \\
\hline n-3/n-6PUFA & 1.1 & 1.08 & 0.92 & 0.58 & 0.57 & 0.83 & 3.15 & 0.39 & 2.97 \\
\hline
\end{tabular}

Values are means of triplicate of nine different feed samples. Values on the same row with different superscripts are significantly different ( $\mathrm{p}<0.05$ ) ${ }^{1}$ Saturated fatty acids consist of: 4:0, 6:0, 8:0, 10:0, 11:0, 12:0, 13:0, 14:0, 15:0, 16:0, 18:0, 20:0 and 24:0

${ }^{2}$ Monounsaturated fatty acids comprise: $14: 1,15: 1,16: 1,18: 1 \mathrm{n} 9 \mathrm{c}, 18: 1 \mathrm{n} 7,20: 1 \mathrm{n} 9$, C22:1n9 and 24:1n9

${ }^{3}$ Polyunsaturated fatty acids consist of: 16:2n4, 16:3n4, 16:4n1, 18:2n6t, 18:2n6c 18:3n4, 18:3n6, 18:3n3, 18:4n3, 20:2, 20:3n6, 20:3n3, 20:5n3, C22:2 and $22: 6$ n 3

n-3 fatty acids were: 18:3n3, 18:4n3; n-6 fatty acids: 18:2n6t, 18:2n6c, 18:3n6; n-3 Long chain polyunsaturated fatty acids found were: $20: 3 \mathrm{n} 3$, 20:5n3 and 22:6n3; n-6 Long chain polyunsaturated fatty acids include: $20: 3 \mathrm{n} 6$

${ }^{4}$ Consisted $18: \ln 9 \mathrm{c}, 20: 1 \mathrm{n} 9, \mathrm{C} 22: \ln 9$ and $24: 1 \mathrm{n} 9$

${ }^{5}$ Comprised: 18:2n6t, 18:2n6c, 18:3n6 and 20:3n6

${ }^{6}$ Included: 18:3n3, 18:4n3, 20:3n3, 20:5n3 and 22:6n3

Table 3. Essential amino acids ( $100 \mathrm{~g}$ crude protein $\left.^{-1}\right)$ of the study diets containing graded levels of S. platensis and C. vulgaris

\begin{tabular}{llllllllll}
\hline Amino acid & Control & SP12.5\% & SP25\% & SP50\% & SP75\% & CL12.5\% & CL25\% & CL50\% & CL75\% \\
\hline Histidine $^{*}$ & $2.02 \pm 0.06^{\mathrm{b}}$ & $2.07 \pm 0.02^{\mathrm{bc}}$ & $2.11 \pm 0.06^{\mathrm{bc}}$ & $2.38 \pm 0.02^{\mathrm{d}}$ & $1.85 \pm 0.00^{\mathrm{a}}$ & $2.01 \pm 0.01^{\mathrm{b}}$ & $2.17 \pm 0.00^{\mathrm{c}}$ & $1.87 \pm 0.00^{\mathrm{a}}$ & $2.00 \pm 0.00^{\mathrm{b}}$ \\
Arginine* $^{\text {Threonine }}$ & $6.47 \pm 0.27^{\mathrm{bc}}$ & $6.57 \pm 0.06^{\mathrm{c}}$ & $6.49 \pm 0.14^{\mathrm{c}}$ & $7.47 \pm 0.09^{\mathrm{d}}$ & $6.29 \pm 0.04^{\mathrm{bc}}$ & $6.10 \pm 0.01^{\mathrm{ab}}$ & $6.30 \pm 0.01^{\mathrm{bc}}$ & $5.76 \pm 0.01^{\mathrm{a}}$ & $6.08 \pm 0.04^{\mathrm{ab}}$ \\
Valine $^{*}$ & $3.68 \pm 0.05^{\mathrm{ab}}$ & $3.78 \pm 0.05^{\mathrm{bc}}$ & $3.79 \pm 0.10^{\mathrm{bc}}$ & $4.43 \pm 0.06^{\mathrm{d}}$ & $3.75 \pm 0.03^{\mathrm{b}}$ & $3.82 \pm 0.02^{\mathrm{bc}}$ & $3.92 \pm 0.00^{\mathrm{c}}$ & $3.57 \pm 0.00^{\mathrm{a}}$ & $3.77 \pm 0.01^{\mathrm{b}}$ \\
Methionine $^{*}$ & $3.64 \pm 0.05^{\mathrm{b}}$ & $3.70 \pm 0.05^{\mathrm{b}}$ & $3.97 \pm 0.09^{\mathrm{c}}$ & $4.14 \pm 0.04^{\mathrm{c}}$ & $3.62 \pm 0.01^{\mathrm{b}}$ & $4.09 \pm 0.20^{\mathrm{c}}$ & $3.23 \pm 0.00^{\mathrm{a}}$ & $3.62 \pm 0.00^{\mathrm{b}}$ & $3.57 \pm 0.00^{\mathrm{b}}$ \\
Lysine $^{*}$ & $2.38 \pm 0.02^{\mathrm{bc}}$ & $2.40 \pm 0.04^{\mathrm{c}}$ & $2.68 \pm 0.05^{\mathrm{e}}$ & $2.91 \pm 0.02^{\mathrm{f}}$ & $2.32 \pm 0.02^{\mathrm{b}}$ & $2.64 \pm 0.01^{\mathrm{de}}$ & $2.57 \pm 0.01^{\mathrm{d}}$ & $2.11 \pm 0.00^{\mathrm{a}}$ & $2.13 \pm 0.00^{\mathrm{a}}$ \\
Isoleucine $^{*}$ & $5.40 \pm 0.14^{\mathrm{c}}$ & $5.09 \pm 0.08^{\mathrm{b}}$ & $6.12 \pm 0.14^{\mathrm{e}}$ & $4.87 \pm 0.09^{\mathrm{b}}$ & $3.87 \pm 0.02^{\mathrm{a}}$ & $6.12 \pm 0.08^{\mathrm{e}}$ & $3.64 \pm 0.01^{\mathrm{a}}$ & $5.71 \pm 0.01^{\mathrm{d}}$ & $4.85 \pm 0.00^{\mathrm{b}}$ \\
Leucine $^{*}$ & $3.24 \pm 0.03^{\mathrm{c}}$ & $3.35 \pm 0.05^{\mathrm{d}}$ & $3.53 \pm 0.07^{\mathrm{e}}$ & $3.80 \pm 0.02^{\mathrm{f}}$ & $3.36 \pm 0.01^{\mathrm{d}}$ & $3.49 \pm 0.01^{\mathrm{e}}$ & $2.83 \pm 0.00^{\mathrm{a}}$ & $3.11 \pm 0.00^{\mathrm{b}}$ & $3.09 \pm 0.00^{\mathrm{b}}$ \\
Phenylalanine/TAA $^{*}$ & $6.43 \pm 0.09^{\mathrm{b}}$ & $6.44 \pm 0.10^{\mathrm{b}}$ & $6.94 \pm 0.15^{\mathrm{c}}$ & $7.18 \pm 0.04^{\mathrm{d}}$ & $6.28 \pm 0.02^{\mathrm{b}}$ & $6.78 \pm 0.00^{\mathrm{c}}$ & $5.62 \pm 0.02^{\mathrm{a}}$ & $6.42 \pm 0.01^{\mathrm{b}}$ & $6.35 \pm 0.00^{\mathrm{b}}$ \\
Tryptophan $^{*}$ & $0.83 \pm 0.07^{\mathrm{a}}$ & $4.04 \pm 0.06^{\mathrm{c}}$ & $4.07 \pm 0.09^{\mathrm{c}}$ & $4.80 \pm 0.00^{\mathrm{e}}$ & $3.95 \pm 0.01^{\mathrm{bc}}$ & $3.97 \pm 0.01^{\mathrm{bc}}$ & $4.28 \pm 0.01^{\mathrm{d}}$ & $3.76 \pm 0.01^{\mathrm{a}}$ & $4.07 \pm 0.00^{\mathrm{c}}$ \\
\hline
\end{tabular}

Values are means of triplicates of nine different feed samples. Mean values on the same row with different superscripts are significantly different $(\mathrm{p}<0.05)$. "Amino acid requirement of C. gariepinus: Histidine - 1.2; Arginine - 3.6; Threonine - 2.8; Valine - 2.4; Methionine A - 2.3; Lysine - 4.8; Isoleucine - 2.0; Leucine - 3.5; Phenylalanine/TAA - 4.0; Tryptophan - 0.5, [Source: Uys (1989); Unprasert. (1984)]. TAA: Total amino acid

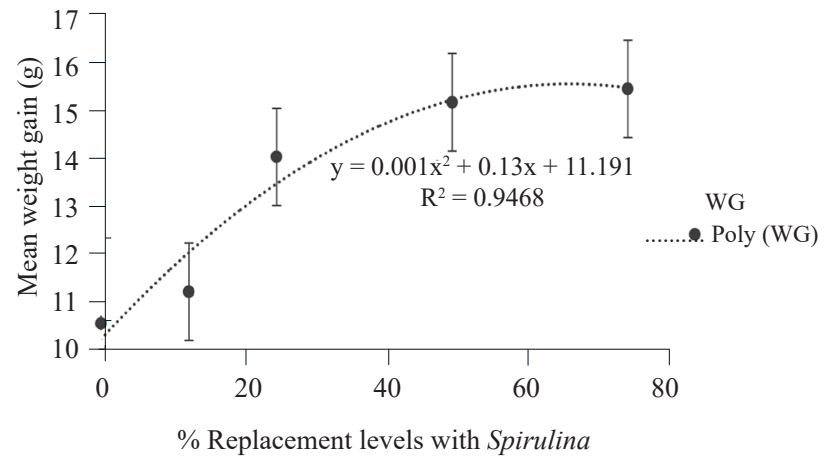

Fig. 1a. Mean weight gain of C. gariepinus fingerlings fed graded levels of Spirulina for 56 days. The optimum level is at $68.5 \%$.

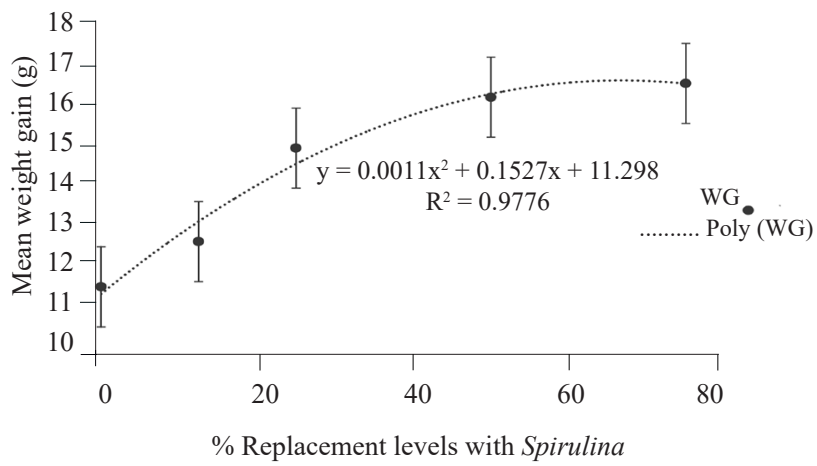

Fig. 1b. Mean weight gain of C. gariepinus fingerlings fed graded levels of Chlorella for 56 days. The optimum level is at $69.4 \%$. 
Table 4. Growth parameters of C. gariepinus fingerlings fed different levels of S. platensis or C. vulgaris diets for 8 weeks

\begin{tabular}{|c|c|c|c|c|c|c|c|c|c|}
\hline Parameter & Control & SP12.5\% & SP25 \% & SP50\% & SP75\% & CL12.5 \% & CL25\% & CL50\% & CL75\% \\
\hline IW (g) & $7.82 \pm 0.06^{\mathrm{a}}$ & $7.88 \pm 0.05^{\mathrm{a}}$ & $7.88 \pm 0.01^{\mathrm{a}}$ & $7.87 \pm 0.03^{\mathrm{a}}$ & $7.85 \pm 0.03^{\mathrm{a}}$ & $7.87 \pm 0.04^{\mathrm{a}}$ & $7.82 \pm 0.00^{\mathrm{a}}$ & $7.89 \pm 0.05^{\mathrm{a}}$ & $7.81 \pm 0.02^{\mathrm{a}}$ \\
\hline FW (g) & $19.29 \pm 0.03^{\mathrm{a}}$ & $19.91 \pm 0.03^{\mathrm{b}}$ & $22.33 \pm 0.01^{\mathrm{d}}$ & $23.30 \pm 0.01^{\mathrm{f}}$ & $23.52 \pm 0.03^{\mathrm{g}}$ & $20.42 \pm 0.04^{\mathrm{c}}$ & $22.60 \pm 0.01^{\mathrm{e}}$ & $23.88 \pm 0.02^{\mathrm{h}}$ & $24.14 \pm 0.01^{\mathrm{i}}$ \\
\hline WG (g) & $11.47 \pm 0.03^{\mathrm{a}}$ & $12.04 \pm 0.09^{\mathrm{b}}$ & $14.45 \pm 0.01^{\mathrm{d}}$ & $15.43 \pm 0.02^{\mathrm{f}}$ & $15.67 \pm 0.01^{\mathrm{g}}$ & $12.55 \pm 0.01^{\mathrm{c}}$ & $14.78 \pm 0.01^{\mathrm{e}}$ & $15.99 \pm 0.05^{\mathrm{h}}$ & $16.32 \pm 0.01^{\mathrm{i}}$ \\
\hline RGR \% & $146.63 \pm 1.63^{\mathrm{a}}$ & $152.90 \pm 2.03^{\mathrm{b}}$ & $183.53 \pm 0.14^{\mathrm{d}}$ & $196.07 \pm 1.05^{\mathrm{f}}$ & $199.69 \pm 0.81^{\mathrm{g}}$ & $159.50 \pm 0.89^{c}$ & $189.01 \pm 0.08^{\mathrm{e}}$ & $202.78 \pm 1.84^{\mathrm{g}}$ & $209.02 \pm 0.61^{\mathrm{h}}$ \\
\hline SGR $\left(\%\right.$ day $\left.^{-1}\right)$ & $2.92 \pm 0.00^{\mathrm{a}}$ & $2.95 \pm 0.00^{\mathrm{b}}$ & $3.07 \pm 0.00^{\mathrm{d}}$ & $3.11 \pm 0.00^{\mathrm{g}}$ & $3.12 \pm 0.00^{\mathrm{f}}$ & $2.98 \pm 0.00^{\mathrm{c}}$ & $3.08 \pm 0.00^{\mathrm{e}}$ & $3.14 \pm 0.00^{\mathrm{h}}$ & $3.15 \pm 0.00^{\mathrm{i}}$ \\
\hline $\mathrm{FI}(\mathrm{g})$ & $17.75 \pm 0.21^{\mathrm{b}}$ & $16.89 \pm 0.08^{\mathrm{ab}}$ & $16.96 \pm 0.02^{\mathrm{ab}}$ & $16.17 \pm 0.01^{\mathrm{a}}$ & $17.92 \pm 0.04^{b}$ & $16.84 \pm 0.02^{\mathrm{ab}}$ & $16.40 \pm 0.01^{\mathrm{a}}$ & $16.20 \pm 0.02^{\mathrm{a}}$ & $16.26 \pm 0.03^{\mathrm{a}}$ \\
\hline FCR & $1.55 \pm 0.01^{\mathrm{i}}$ & $1.40 \pm 0.02^{\mathrm{h}}$ & $1.17 \pm 0.01^{\mathrm{f}}$ & $1.05 \pm 0.01^{\mathrm{c}}$ & $1.14 \pm 0.01^{\mathrm{e}}$ & $1.34 \pm 0.01^{\mathrm{g}}$ & $1.11 \pm 0.13^{\mathrm{d}}$ & $0.95 \pm 0.01^{\mathrm{b}}$ & $0.93 \pm 0.01^{\mathrm{a}}$ \\
\hline PI & $8.49 \pm 0.01^{\mathrm{g}}$ & $8.52 \pm 0.00^{\mathrm{g}}$ & $7.93 \pm 0.00^{\mathrm{e}}$ & $7.37 \pm 0.00^{c}$ & $8.17 \pm 0.02^{\mathrm{f}}$ & $7.60 \pm 0.01^{\mathrm{d}}$ & $7.14 \pm 0.01^{\mathrm{b}}$ & $7.04 \pm 0.03^{\mathrm{a}}$ & $7.03 \pm 0.00^{\mathrm{a}}$ \\
\hline PER & $1.35 \pm 0.01^{\mathrm{a}}$ & $1.41 \pm 0.02^{\mathrm{b}}$ & $1.82 \pm 0.01^{\mathrm{d}}$ & $2.09 \pm 0.01^{\mathrm{g}}$ & $1.92 \pm 0.01^{\mathrm{e}}$ & $1.65 \pm 0.01^{\mathrm{c}}$ & $2.07 \pm 0.01^{\mathrm{f}}$ & $2.27 \pm 0.01^{\mathrm{h}}$ & $2.33 \pm 0.01^{\mathrm{i}}$ \\
\hline PPV & $58.19 \pm 0.09^{\mathrm{d}}$ & $83.61 \pm 0.04^{\mathrm{i}}$ & $78.84 \pm 0.10^{\mathrm{h}}$ & $46.80 \pm 0.01^{\mathrm{c}}$ & $36.24 \pm 0.07^{\mathrm{a}}$ & $60.39 \pm 0.06^{\mathrm{e}}$ & $39.73 \pm 0.03^{b}$ & $75.55 \pm 0.33^{g}$ & $72.31 \pm 0.12^{\mathrm{f}}$ \\
\hline K Factor & $1.39 \pm 0.00^{\mathrm{a}}$ & $1.50 \pm 0.03^{\mathrm{b}}$ & $1.66 \pm 0.03^{\mathrm{d}}$ & $1.69 \pm 0.02^{\mathrm{de}}$ & $1.73 \pm 0.02^{\mathrm{e}}$ & $1.58 \pm 0.01^{\mathrm{g}}$ & $1.67 \pm 0.01^{\mathrm{f}}$ & $1.84 \pm 0.01^{\mathrm{g}}$ & $1.89 \pm 0.01^{\mathrm{h}}$ \\
\hline Survival (\%) & $86.67 \pm 8.82^{\mathrm{a}}$ & $96.67 \pm 3.33^{\mathrm{a}}$ & $96.67 \pm 3.33^{\mathrm{a}}$ & $93.33 \pm 3.33^{\mathrm{a}}$ & $100.00 \pm 0.0^{\mathrm{a}}$ & $96.67 \pm 3.33^{\mathrm{a}}$ & $93.33 \pm 3.33^{\mathrm{a}}$ & $96.67 \pm 3.33^{\mathrm{a}}$ & $96.67 \pm 3.3^{\mathrm{a}}$ \\
\hline
\end{tabular}

Values are means of a triplicate of ten fish. Mean values on the same row with different superscripts are significantly different (p<0.05). SGR: Specific growth Rate; FCR: Feed conversion ratio; RGR: Relative growth rate; PI: Protein intake; PER: Protein efficiency ratio; K: Fulton's condition factor; PPV: Protein productive value.

Table 5 clearly shows significant $(\mathrm{p}<0.05)$ differences in the experimental fish carcass composition compared to the control group. The mean protein value $(61.97 \% \pm 010)$ recorded for SP12.5\% was significantly $(\mathrm{p}<0.05)$ higher than all other experimental fish groups except for SP25\%. Crude lipid $(14.81 \% \pm 0.08)$ and gross energy (20.81 $\left.\mathrm{KJ} \mathrm{g}^{-1} \pm 1.65\right)$ were significantly $(\mathrm{p}<0.05)$ higher in SP75\% than other diets, whereas ash, moisture and NFE were significantly $(\mathrm{p}<0.05)$ lower in $\mathrm{SP} 75 \%$, control and SP25\% respectively.

\section{Discussion}

This study investigated the effect of $S$. platensis and C. vulgaris as partial replacement for fishmeal protein on growth and body compositions of African catfish (C.gariepinus) fingerlings. The basal diet was replaced with 0 to $75 \%$ S. platensis and C. vulgaris. The findings from this study showed that experimental diets contained similar fatty acids, essential amino acids and gross energy, relative to the control diet, although the two microalgae varied in their chemical composition of essential amino acid and fatty acid profiles. The crude protein and lipid values of the experimental diets fall within the range recommended for optimal growth of catfish fingerlings (FAO, 2009). The experimental diets contained high quantity of amino acids which are greater than values reported by Jimoh et al. (2014) for sesame seed meal fed to African catfish and slightly higher than values recommended for African catfish by Unprasert (1994), except for the values obtained for methionine in CL75\%, lysine in SP75 and CL25\%. Cysteine was however not determined, as Lovell (1989) reported that non-EAAs like cysteine and tyrosine could replace about 60 and $50 \%$ catfish requirements for methionine and phenylalanine, respectively.

Fatty acids (FAs) are imperative constituents of biomembranes of fish besides providing a premise of vitality (Parrish et al., 2012). In many fish species, eicosapentaenoic acid (EPA) and arachidonic acid (ARA) are essential for vital metabolic functions (Tocher, 2015). Microalgae are the principal producers of FAs. Fish, for the most part, require linolenic acid (18:3n-3) for optimal

Table 5. Proximate composition (\% dry weight) of the whole body of C. gariepinus fed S. platensis and C. vulgaris diets

\begin{tabular}{lllllll}
\hline & \multicolumn{5}{c}{ Composition } \\
\cline { 2 - 7 } Treatments & Crude protein & Crude lipid & Ash & Moisture & NFE & Gross energy \\
Initial & $54.85 \pm 0.09^{\mathrm{a}}$ & $7.16 \pm 0.00^{\mathrm{a}}$ & $20.95 \pm 0.04^{\mathrm{h}}$ & $5.00 \pm 0.03^{\mathrm{e}}$ & $12.06 \pm 0.08^{\mathrm{d}}$ & $17.81 \pm 0.20^{\mathrm{a}}$ \\
Control & $59.79 \pm 0.00^{\mathrm{d}}$ & $10.21 \pm 0.03^{\mathrm{c}}$ & $17.71 \pm 0.11^{\mathrm{d}}$ & $2.83 \pm 0.01^{\mathrm{a}}$ & $9.48 \pm 0.08^{\mathrm{c}}$ & $19.75 \pm 0.56^{\mathrm{d}}$ \\
SP12.5\% & $61.97 \pm 0.10^{\mathrm{e}}$ & $7.53 \pm 0.01^{\mathrm{b}}$ & $18.39 \pm 0.10^{\mathrm{f}}$ & $4.04 \pm 0.00^{\mathrm{d}}$ & $8.07 \pm 0.01^{\mathrm{b}}$ & $18.98 \pm 0.49^{\mathrm{b}}$ \\
SP25\% & $61.10 \pm 0.40^{\mathrm{e}}$ & $10.16 \pm 0.00^{\mathrm{c}}$ & $18.82 \pm 0.00^{\mathrm{g}}$ & $3.99 \pm 0.01^{\mathrm{d}}$ & $5.95 \pm 0.38^{\mathrm{a}}$ & $19.45 \pm 0.72^{\mathrm{c}}$ \\
SP50\% & $58.30 \pm 0.25^{\mathrm{c}}$ & $12.14 \pm 0.03^{\mathrm{d}}$ & $17.62 \pm 0.01^{\mathrm{d}}$ & $3.92 \pm 0.01^{\mathrm{d}}$ & $8.04 \pm 0.22^{\mathrm{b}}$ & $19.92 \pm 0.26^{\mathrm{e}}$ \\
SP75\% & $57.81 \pm 0.40^{\mathrm{b}}$ & $14.81 \pm 0.08^{\mathrm{g}}$ & $16.08 \pm 0.05^{\mathrm{a}}$ & $3.61 \pm 0.19^{\mathrm{bc}}$ & $7.71 \pm 0.34^{\mathrm{b}}$ & $20.81 \pm 1.65^{\mathrm{g}}$ \\
CL12.5\% & $59.44 \pm 1.07^{\text {cd }}$ & $13.30 \pm 0.15^{\mathrm{f}}$ & $16.78 \pm 0.06^{\mathrm{bc}}$ & $3.47 \pm 0.00^{\mathrm{b}}$ & $7.03 \pm 0.98^{\mathrm{ab}}$ & $20.50 \pm 0.96^{\mathrm{f}}$ \\
CL25\% & $57.68 \pm 0.17^{\mathrm{b}}$ & $12.55 \pm 0.11^{\mathrm{e}}$ & $18.04 \pm 0.00^{\mathrm{e}}$ & $3.63 \pm 0.03^{\mathrm{bc}}$ & $8.11 \pm 0.03^{\mathrm{b}}$ & $19.95 \pm 0.20^{\mathrm{e}}$ \\
CL50\% & $60.17 \pm 0.24^{\text {de }}$ & $7.16 \pm 0.00^{\mathrm{a}}$ & $16.93 \pm 0.06^{\mathrm{c}}$ & $4.07 \pm 0.03^{\mathrm{d}}$ & $11.69 \pm 0.14^{\mathrm{d}}$ & $19.01 \pm 0.75^{\mathrm{b}}$ \\
CL75\% & $59.93 \pm 0.57^{\mathrm{d}}$ & $10.13 \pm 0.03^{\mathrm{c}}$ & $16.54 \pm 0.01^{\mathrm{b}}$ & $3.71 \pm 0.03^{\mathrm{c}}$ & $9.71 \pm 0.50^{\mathrm{c}}$ & $19.79 \pm 0.93^{\mathrm{d}}$
\end{tabular}

Values are means of triplicate groups of 3 fish per replicate. Mean values in the same column with different superscripts are significantly different $(\mathrm{p}<0.05)$. 
growth. African catfish has the ability to change dietary linolenic acid to EPA (20:5n-3) which is sufficient to meet its metabolic demands (Tucker and Hargreaves, 2004). The n-3 EFA prerequisite could be met by either 1 to $2 \%$ linolenic acid or 0.5 to $0.75 \%$ n-3 HUFAs (Satoh et al., 1989), which can be supplied by supplementing marine fish oil, for example, menhaden oil in the diet (Morris et al., 1995; Manning et al., 2007). This can be likened to the palm oil supplement in the experimental diets. However, fatty acids play vital roles in the development of catfish. The EFA requirements of $C$. gariepinus according to Uys and Hecht (1985) are linoleic acid (18:2n6), arachidonic $(20: 4 n-6)$, linolenic $(18: 3 n 3)$, eicosapentaenoic $(20: 5 n 3)$ and docosahexaenoic $(22: 6 n 3)$ acids in the ratio $1: 1$. The values for linoleic, linolenic, eicosapentaenoic and docosahexaenoic acids obtained in the present study are higher than the values obtained for African catfish by Li et al. (2009) from dried Schizochytrium sp. diets.

The outcome of this study shows that $C$. gariepinus fingerlings readily accepted the experimental diets as they exhibited good growth. This study further reveals that replacing fishmeal with $C$. vulgaris, positively correlated to the growth of the fingerlings resulting in higher weight gain and SGR. This might be as a result of growth factor in Chlorella sp. (Bengwayan et al., 2010), while higher PER may be due to high protein digestibility. C. vulgaris diets (50 and 75\%) have higher CP compared to other CL treatments and this high protein could induce the fish to use protein as energy source resulting in significant weight gain. Optimum weight gain was achieved at $69.4 \%$ replacement of FM with $C$. vulgaris and higher substitution beyond this could not provide a further increase in weight gain. Increasing the replacement level of fishmeal with Chlorella sp. above 50\% did not improve the growth performance of Nile tilapia (Badwy et al., 2008). Conversely, Khani et al. (2017) showed that optimum growth performance of Koi carp is best attained at 5\% C. vulgaris replacement of fishmeal.

In addition, previous studies have attributed the comparable growth performance associated with Chlorella diets, to growth promoters such as sufficient amounts of macro nutrients and Chlorella growth factors, present in C. vulgaris (Yamaguchi, 1996; Badwy et al., 2008). More so, the growth performance associated with dietary Chlorella could be atributed to high digestibility of microalgae and possession of significant concentration of polysaccharides, lipids, minerals and other bioactive compounds involved in physiological activities ( $\mathrm{Xu}$ et al., 2014; Khani et al., 2017). Khani et al. (2017) suggested that Chlorella diets increase the digestive enzymes in the pancreas and intestine of Koi carp, thereby enhancing the diet utilisation rate through increase in the activity of digestive enzymes. Another study by the author on nutrient digestibility of dietary Chlorella showed that it was better digested and assimilated than the commercial diet.

The present study also showed that $S$. platensis diets improve growth performance of $C$. gariepinus fingerlings as indicated by the weight gain, FCR, PER and SGR. As the Spirulina inclusion level increased, the body weight gain of the fingerlings also increased, although this increase showed an indirect relationship with the CP of Spirulina diets. The findings here show that dietary Spirulina at the same inclusion level (50:50) with FM gives better PER, which suggests that the diet is well digested and assimilated. It has been suggested that S. platensis improves the intestinal flora in fish, thereby allowing a breakdown of ingestible feed components to extract more nutrients and stimulates the production of enzymes that transport fats for metabolisms instead of storage within the fish (James et al., 2006). Abdel-Tawwab and Ahmad (2009) stated that the role Spirulina plays in the digestibility of nutrients and its numerous vitamins and minerals is responsible for its positive effects on fish development. The present study agrees with the works of Nandeesha et al. (1998) and Palmegiano et al. (2005) who successfully replaced FM with up to 60 and $100 \%$ dietary FM of C. carpio and Siberian sturgeon (Acipenser baeri) respectively, with no negative consequence.

Although some studies had recommended partial $(0.5-5 \%)$ replacement of fishmeal with $S$. platensis for optimal growth performance (Abdel-Tawwab and Ahmad, 2009; Promya and Chitmanat, 2011), a total replacement $(100 \%)$ was reported to show improved growth performance. These studies also suggested that major inclusion $(25-100 \%)$ of dietary Spirulina significantly increased the digestive enzymes activity of common carp, rohu and catla (Nandeesha et al., 1994; Umesh et al., 1994; Nandeesha et al., 1998). More importantly, the cellular structure of Spirulina lacks cellulose and is thus, easily digestible (Promya and Chitmanat, 2011). Similarly, Teuling et al. (2017) reported that protein apparent digestibility coefficient of $C$. vulgaris in C. gariepinus is about $80 \%$ and nutrient digestibility of the algae does not relate to its mechanical cell wall hardness.

The optimum replacement of fishmeal with SP and CL was 68.5 and $69.4 \%$, respectively. However, any further increase in the inclusion level of these algae beyond the optimum will not improve the growth of C. gariepinus fingerlings further and this has been previously reported for catla (Catla catla) by Nandeesha et al. (2001). These variabilities obtained in growth response of fishes to dietary Spirulina and other algal species in the different studies visibly revealed that the growth response to algae might be species-specific. Other 
possible factors are variability in the nutrient composition, inclusion level of Spirulina or other algae as well as the nutritional contents of the test diets (Nandeesha et al., 1998; Olvera-Novoa et al., 1998; Nandeesha etal., 2001; Takeuchi et al., 2002). Olvera-Novoa et al. (1998) suggested that the harmful effects of inclusion of high levels of Spirulina in the diet on fish growth are the decline in phosphorous accessibility and reduction in feed palatability. This call for further study on the prolonged effect of extended feeding of C. gariepinus with high inclusion levels of algae.

Fulton's (K) factor provides reliable information on the actual state of health, growth and well-being of fish (Araneda et al., 2008). The present study reveals that both C. vulgaris and S. platensis diets showed better Fulton's condition factor and survival rate when compared to the fish fed control diet. This further corroborates the high growth performance exhibited by fish fed algal diets and hence, it can be suggested that together these factors signify the good health condition of the fish.

The results obtained in this study showed that there is a positive correlation between the dietary protein and the fish muscle CP. The whole body composition of C. gariepinus fed dietary Chlorella and Spirulina, shows higher CP than the control. This could be due to the higher protein productive values observed with the algal diets, which signify good assimilation of the protein. It has been suggested that fish do not have a specific protein requirement, but a definite requirement for essential amino acids (EAA) content of the protein (Miles and Chapman, 2007). This is due to the fact that the dietary proteins will be digested and broken down into amino acids, which can be used efficiently for maintenance, health and synthesis of worn out tissues and thus, result in maximum feed efficiency and growth (Miles and Chapman, 2007). This assertion is supported by the profiles of EAA exhibited by the algal diets as observed in the present study.

In this work, protein levels of fish carcass decreased as the inclusion of $S$. platensis increased while fat increased in a dose dependent manner. The high fat and energy levels recorded in some of the carcasses might be because of varied inclusion levels of the palm oil which was mixed with the fish oil. This corroborates the findings of researchers who opined that when plant proteins are included in the diets of fish, there is potential increase in fish lipid (Yildirim-Aksoy et al., 2007; Bake et al., 2016). The findings in this study are consistent with other studies, which found Spirulina to increase fat deposition (Atack et al., 1979; Watanabe, 1990; Nandeesha et al., 1998; 2001). However, it differs from that of C. carpio where a significant decrease occurred in body lipid composition by similar Spirulina administration. This is due to the effects of different Spirulina species on fat deposition (Nandeesha et al., 1998; Nandeesha et al., 2001). Abdel-Tawwab and Ahmad (2009) stated that the influence of Spirulina on body lipid as well as protein are linked with their production plus build-up level, as well as the growth rate of the animal. Chlorella fed fish shows decrease in fat deposit in flesh with increase in inclusion levels in the diets. This could be due to the high quantity of polyunsaturated fatty acids in Chlorella in this study or due to the presence $\beta$ - 1,3 -glucan, a strong immune booster, free-radical forager and reducer of blood fat. Chlorella had superior activity in hindering lipid peroxidation compared to glutathione and has antioxidant properties (Bengwayan et al., 2010).

There was a positive correlation between energy in the carcass and dietary lipid. Lipids are important sources of energy (Webster and Lim, 2002) as they have more energy per unit weight than any other organic compound; one gram of fat comprises about two folds as much aggregate energy as a single gram of protein or carbohydrate (Guillaume et al., 2001). Previous researchers have shown that considerable use of vegetable oil as an energy source in fish diets produced growth response in fish (Babalola and Adebayo, 2007; Aderolu and Akinremi, 2009). Fish oil and palm oil at ratio of 1:3 were used in this study and the results obtained have demonstrated that they are good energy source and had no palatability problem. Previously, the inclusion of vegetable oils in the diet of seabream was found to promote fat build up in the liver (Caballero et al., 2004). However, there seems to be no direct relationship between energy in the carcass and diets. This implies that energy in the carcass might have been derived from different components of the diets, mostly lipids.

This study determined the effect of partial substitution of fishmeal by $S$. platensis and C. vulgaris to assess the practicability of their use in $C$. gariepinus feed. Findings from this study revealed that SP and CL can optimally replace fishmeal up to $68 \%$. Essential nutrients such as amino acids and fatty acids found in fishmeal are also abundantly present in SP and CL diets, while the carcass composition of fish fed SP and CL diets showed higher crude protein and energy compared to fishmeal fed carcass. Thus, findings from this study suggest that both $S$. platensis and $C$. vulgaris could substitute fishmeal in C. gariepinus diets. Nonetheless, further studies are still required to evaluate the long term effects of feeding C. gariepinus with dietary S. platensis and C. vulgaris from fingerlings to adult. The ability of both algae to thrive in different growth media would reduce feed cost involved in aquaculture production. Globally, fishmeal and oil production are declining and hence, Chlorella and Spirulina could play an important role to supply not only protein but EAAs and EFAs to $C$. gariepinus as they are sustainable sources of these products. However, large scale 
production of these algae through advanced technology is recommended. This would serve as a sustainable source of protein for aquaculture and would help to curtail the over dependence and competition between the aquaculture and other livestock sectors on fishmeal.

\section{Acknowledgments}

We thank University Malaya for financial support through grants FP049-2014A and RPO15G-14AFR. Our appreciation also goes to the staff members of the Fisheries Research Institute, Department of Fisheries, Glami Lemi Jelebu Malaysia, the Algae Research Laboratory and Academic Development Centre (ADEC), University Malaya for technical support and statistical analysis.

\section{References}

Abdel-Tawwab, M. and Ahmad, M. H. 2009. Live spirulina (Arthrospira platensis) as a growth and immunity promoter for Nile tilapia, Oreochromis niloticus (L.), challenged with pathogenic Aeromonas hydrophila. Aquac. Res., 40(9): 1037-1046. https://doi.org/10.1111/j.13652109.2009.02195.x.

Abdulrahman, N. M. and Ameen, H. J. H. 2013. The effect of replacing fishmeal with spirulina on microbial load in common carp Cyprinus carpio L. Iraq. J. Vet. Sci., 27(1): $57-60$

Abdulrahman, N. M. and Ameen, H. J. H. 2014. Replacement of fishmeal with microalgae spirulina on common carp weight gain, meat and sensitive composition and survival. Pak. J. Nutr., 13(2): 93. https://doi.org/10.3923/pjn.2014.93.98.

Aderolu, A. Z. and Akinremi, O. A. 2009. Dietary effects of coconut oil and peanut oil in improving biochemical characteristics of Clarias gariepinus juvenile. Turk. J. Fish. Aquat. Sci., 9(1): 105-110.

Al-Dohail, M. A., Hashim, R. and Aliyu-Paiko, M. 2009. Effects of the probiotic Lactobacillus acidophilus, on the growth performance, haematology parameters and immunoglobulin concentration in African catfish (Clarias gariepinus, Burchell 1822) fingerling. Aquac. Res., 40(14): 1642-1652. https://doi.org/10.1111/j.13652109.2009.02265.x.

Araneda, M., Perez, E. P. and Gasca-Leyva, E. 2008. White shrimp Penaeus vannamei culture in freshwater at three densities: condition state based on length and weight. Aquaculture, 283(1-4): 13-18. https://doi.org/10.1016/j. aquaculture.2008.06.030.

Atack, T., Jauncey, K. and Matty, A. J. 1979. The utilisation of some single cell proteins by fingerling mirror carp (Cyprinus carpio). Aquaculture, 18(4): 337-348. https:// doi.org/10.1016/0044-8486(79)90037-1.

Babalola, T. O. O. and Adebayo, M. 2007. Effect of dietary lipid level on growth performance and feed utilisation by Heterobranchus longifilis fingerlings. J. Fish. Int., 2(1): 60-64.
Badwy, T. M., Ibrahim, E. and Zeinhom, M. 2008. Partial replacement of fishmeal with dried microalga (Chlorella spp. and Scenedesmus spp.) in Nile tilapia (Oreochromis niloticus) diets. Proceedings of the $8^{\text {th }}$ International Symposium on Tilapia in Aquaculture, Ministry of Agriculture, Cairo, Egypt, p. 801-811.

Bake, G. G., Yusuf, I. and Sadiku, S. O. E. 2016. Evaluation and nutrient quality of toasted flamboyant seed (Delonix regia) meal in the diet of Clarias gariepinus fingerlings. J. Agric. Ecol. Res. Int., 5(2): 1-9. https://doi.org/10.9734/ jaeri/2016/21687.

Becker, E. 2007. Micro-algae as a source of protein. Biotechnol. $A d v$., 25(2): 207-210. https://doi.org/10.1016/j.biotechadv. 2006.11.002.

Belay, A. 2002. The potential application of spirulina (Arthrospira) as a nutritional and therapeutic supplement in health management. J. Am. Nutraceut. Ass., 5: 27-48.

Bengwayan, P. T., Laygo, J. C., Pacio, A. E., Poyaoan, J. L. Z., Rebugio, J. F. and Yuson, A. L. L. 2010. A comparative study on the antioxidant property of Chlorella (Chlorella sp.) tablet and glutathione tablet. E-int. Sci. Res. J., 2 (1): 25-35.

Caballero, M., Izquierdo, M., Kjorsvik, E., Fernandez, A. and Rosenlund, G. 2004. Histological alterations in the liver of seabream, Sparus aurata L., caused by short-or longterm feeding with vegetable oils. Recovery of normal morphology after feeding fish oil as the sole lipid source. J. Fish Dis., 27(9): 531-541. https://doi.org/10.1111/j.1365 $-2761.2004 .00572 . x$.

Dalle Zotte, A., Sartori, A., Bohatir, P., Remignon, H. and Ricci, R. 2013. Effect of dietary supplementation of spirulina (Arthrospira platensis) and Thyme (Thymus vulgaris) on growth performance, apparent digestibility and health status of companion dwarf rabbits. Livestock Sci., 152(2-3): 182-191. https://doi.org/10.1016/j.livsci.2012.12.017.

Duncan, D. B. 1955. Multiple range and multiple F tests. Biometrics, 11(1): 1-42. https://doi.org/10.2307/3001478

FAO 2009. Fishstat Plus: Universal software for fishery statistical time series. Aquaculture production 1950-2007. Fisheries Department, F. I., Data and Statistics Unit, Food and Agricultural Organisation, Rome, Italy.

Fasakin, E. A., Balogun, A. M. and Ajayi, O. O. 2003. Evaluation of full-fat and defatted maggot meals in the feeding of clariid catfish Clarias gariepinus fingerlings. Aquac. Res., 34(9): 733-738. https://doi.org/10.1046/j.1365-2109. 2003.00876.x

Gatlin, D. M. 2007. Dietary supplements for the health and quality of cultured fish. CABI Publishing, Willingford, UK.

Goda, A., El-Haroun, E. and Kabir Chowdhury, M. 2007. Effect of totally or partially replacing fish meal by alternative protein sources on growth of African catfish Clarias gariepinus (Burchell, 1822) reared in concrete tanks. Aquac. Res., 38(3): 279-287. https://doi.org/10.1111/j. 1365-2109.2007.01663.x. 
Gouveia, L., Batista, A. P., Isabel, S., Raymundo, A. and Bandarra, N. M. 2008. Microalgae in novel food products. In: Konstantinos, N. and Papadopoulos, P. (Eds.), Food chemistry research developments. Nova Science Publishers Inc., New York, USA.

Guillaume, J., Kaushik, S., Bergot, P. and Metailler, R. 2001. Nutrition and feeding of fish and crustaceans, Springer, Berlin, Germany.

Guroy, D., Guroy, B., Merrifield, D., Ergun, S., Tekinay, A. and Yigit, M. 2011. Effect of dietary Ulva and Spirulina on weight loss and body composition of rainbow trout, Oncorhynchus mykiss (Walbaum), during a starvation period. J. Anim. Physiol. Anim. Nutr., 95 (3): 320-327. https://doi.org/10.1111/j.1439-0396.2010.01057.x.

Heinrikson, R. L. and Meredith, S. C. 1984. Amino acid analysis by reverse-phase high-performance liquid chromatography: precolumn derivatization with phenylisothiocyanate. Anal. Biochem., 136(1): 65-74.https://doi.org/10.1016/0003-2697 (84)90307-5.

Htun-Han, M. 1978. The reproductive biology of the dab Limanda limanda (L.) in the North Sea: Gonosomatic index, hepatosomatic index and condition factor. J. Fish Biol., 13(3): 369-378.

Ichihara, K. I. and Fukubayashi, Y. 2010. Preparation of fatty acid methyl esters for gas-liquid chromatography. J. Lipid Res., 51(3): 635-640. https://doi.org/10.1194/jlr.d001065.

James, R., Sampath, K., Thangarathinam, R. and Vasudevan, I. 2006. Effect of dietary spirulina level on growth, fertility, coloration and leucocyte count in red swordtail, Xiphophorus helleri. Isr. J. Aquac. Bamidgeh. 58(2): 97-104.

Jimoh, W., Fagbenro, O. and Adeparusi, E. 2014. Response of African catfish, Clarias gariepinus (Burchell 1822), fingerlings fed diets containing differently timed wet-heattreated sesame (Sesamum indicum) seedmeal. Agric. Sci., 5(12): 1159. https://doi.org/10.4236/as.2014.512126.

Khani, M., Soltani, M., Shamsaie Mehrjan, M., Foroudi, F. and Ghaeni, M. 2017. The effects of Chlorella vulgaris supplementation on growth performance, blood characteristics and digestive enzymes in koi (Cyprinus carpio). Iran. J. Fish. Sci., 16(2): 832-843.

Kim, S.- S., Rahimnejad, S., Kim, K.- W. and Lee, K. -J. 2013. Partial replacement of fish meal with Spirulina pacifica in diets for parrot fish (Oplegnathus fasciatus). Turk. J. Fish. Aquat. Sci., 13 (2): 197-204. https://doi.org/10.4194/13032712-v13 201.

Lehane, S. 2013. Fish for the future: aquaculture and food security.Strategic Analysis Paper, Future Directions International. http://www. futuredirections. org. au/ publication/fish-forthe-future-aquaculture-and-food security (Accessed 12 July 2017).

Li, M. H., Robinson, E. H., Tucker, C. S., Manning, B. B. and Khoo, L. 2009. Effects of dried algae Schizochytrium sp., a rich source of docosahexaenoic acid on growth, fatty acid composition and sensory quality of channel catfish Ictalurus punctatus. Aquaculture, 292(3-4): 232-236. https://doi.org/10.1016/j.aquaculture.2009.04.033.

Lordan, S., Ross, R. P. and Stanton, C. 2011. Marine bioactives as functional food ingredients: potential to reduce the incidence of chronic diseases. Mar. Drugs, 9(6): 1056-1100. https://doi.org/10.3390/md9061056.

Lovell, T. 1989. Feed formulation and processing. In: Nutrition and feeding of fish. Springer, Boston, USA, p. 107-127.

Manning, B. B., Li, M. H. and Robinson, E. H. 2007. Feeding channel catfish Ictalurus punctatus, diets amended with refined marine fish oil elevates Omega-3 highly unsaturated fatty acids in fillets. J. World Aquac. Soc., 38(1): 49-58. https://doi.org/10.1111/j.1749-7345.2006.00072.x.

Miles, R. D. and Chapman, F. A. 2007. The concept of ideal protein in formulation of aquaculture feeds. University of Florida IFAS Extension, p. 1-3.

Mirza, Y. 2004. Winfeed 2.8. Winfeed LTD., Cambridge, UK.

Morris, C., Haynes, K., Keeton, J. and Gatlin, D. 1995. Fish oil dietary effects on fatty acid composition and flavour of channel catfish. J. Food Sci., 60(6): 1225-1227. https://doi. org/10.1111/j.1365-2621.1995.tb04561.x.

Nandeesha, M., De Silva, S., Murthy, D. K. and Dathatri, K. 1994. Use of mixed feeding schedules in fish culture: field trials on catla, Catla catla (Hamilton-Buchanan), rohu, Labeo rohita (Hamilton) and common carp, Cyprinus carpio L. Aquac.. Res., 25(6): 659-670. https://doi.org/ 10.1111/j.1365-2109.1994.tb00730.x.

Nandeesha, M., Gangadhar, B., Varghese, T. and Keshavanath, P. 1998. Effect of feeding Spirulina platensis on the growth, proximate composition and organoleptic quality of common carp, Cyprinus carpio L. Aquac. Res., 29(5): 305-312. https://doi.org/10.1111/j.1365-2109.1998.tb01135.x.

Nandeesha, M., Gangadhara, B., Manissery, J. and Venkataraman, L. 2001. Growth performance of two Indian major carps, catla (Catla catla) and rohu (Labeo rohita) fed diets containing different levels of Spirulina platensis. Bioresour. Technol., 80(2): 117-120. https://doi.org/10.1016/s09608524(01)00085-2.

Nielsen, H. K. and Hurrell, R. F. 1985. Tryptophan determination of food proteins by HPLC after alkaline hydrolysis. J. Sci. Food Agric., 36(9): 893-907.

NRC 2011. Nutrient requirements of fish and shrimp, National Academies Press, USA, 399 pp. https://doi.org/ 10.17226/13039.

Oliva-Teles, A. and Gonçalves, P. 2001. Partial replacement of fishmeal by brewers yeast (Saccaromyces cerevisae) in diets for seabass (Dicentrarchus labrax) juveniles. Aquaculture, 202(3-4): 269-278. https://doi.org/10.1016/ s0044-8486(01)00777-3. 
Olvera-Novoa, M., Dominguez-Cen, L., Olivera-Castillo, L. and Martinez-Palacios, C. A. 1998. Effect of the use of the microalga Spirulina maxima as fish meal replacement in diets for tilapia, Oreochromis mossambicus (Peters) fry. Aquac. Res., 29(10): 709-715. https://doi.org/10.1046/j. 1365-2109.1998.29100709.x.

Otleş, S. and Pire, R. 2001. Fatty acid composition of Chlorella and Spirulina microalgae species. J. AOAC Int., 84(6): 1708-1714.

Palmegiano, G. B., Agradi, E., Forneris, G., Gai, F., Gasco, L., Rigamonti, E., Sicuro, B. and Zoccarato, I. 2005. Spirulina as a nutrient source in diets for growing sturgeon (Acipenser baeri). Aquac. Res., 36(2): 188-195. https:// doi.org/10.1111/j.1365-2109.2005.01209.x.

Parrish, C. C., French, V. M. and Whiticar, M. J. 2012. Lipid class and fatty acid composition of copepods (Calanus finmarchicus, C. glacialis, Pseudocalanus sp., Tisbe furcata and Nitokra lacustris) fed various combinations of autotrophic and heterotrophic protists. J. Plankton Res., 34(5): 356-375. https://doi.org/10.1093/plankt/fbs003.

Phang, S., Miah, M., Yeoh, B. and Hashim, M. 2000. Spirulina cultivation in digested sago starch factory wastewater. J. Appl. Phycol., 12(3-5): 395-400. https://doi.org/10.1023/ a:1008157731731.

Pradhan, J. and Das, B. K. 2015. Effect of dietary Chlorella vulgaris on liver enzymatic profiles of rohu Labeo rohita (Hamilton, 1822). Indian J. Fish., 62(2): 132-136.

Promya, J. and Chitmanat, C. 2011. The effects of Spirulina platensis and Cladophora algae on the growth performance, meat quality and immunity stimulating capacity of the African sharptooth catfish (Clarias gariepinus). Int. J. Agric. Biol., 13(1): 77-82.

Qihuan, Z., Ming, Q., Wei, X., Zhen, G., Rong, S. and Zhitao, Q. 2014. Effects of dietary administration of Chlorella on the immune status of gibel carp, Carassius auratus gibelio. Ital. J. Anim. Sci., 13: 653-656. https://doi.org/10.4081/ijas. 2014.3168.

Reyes-Becerril, M., Guardiola, F., Rojas, M., Ascencio-Valle, F. and Esteban, M. A. 2013. Dietary administration of microalgae Navicula sp. affects immune status and gene expression of gilthead seabream (Sparus aurata). Fish Shellfish Immunol., 35(3): 883-889. https://doi.org/10.1016/j.fsi. 2013.06.026.

Richmond, A. 2004. Handbook of microalgal culture: biotechnology and applied phycology, Wiley Online Library. https://doi.org/ 10.1023/b:japh.0000044943.45511.5e.

Satoh, S., Poe, W. E. and Wilson, R. P. 1989. Effect of dietary n-3 fatty acids on weight gain and liver polar lipid fatty acid composition of fingerling channel catfish. J. Nutr., 119(1): 23-28. https://doi.org/10.1093/jn/119.1.23.

Sayed, A. E.-D. H. and Fawzy, M. A. 2014. Effect of dietary supplementation of Spirulina platensis on the growth and haematology of the catfish Clarias gariepinus. J. Adv. Biol., 5(2): 625-635.
Schulz, C., Knaus, U., Wirth, M. and Rennert, B. 2005. Effects of varying dietary fatty acid profile on growth performance, fatty acid, body and tissue composition of juvenile pike perch (Sander lucioperca). Aquac. Nutr., 11(6): 403-413. https://doi.org/10.1111/j.1365-2095.2005.00369.x.

Spolaore, P., Joannis-Cassan, C., Duran, E. and Isambert, A. 2006. Commercial applications of microalgae. J. Biosci. Bioeng., 101(2): 87-96. https://doi.org/10.1263/jbb.101.87.

Takeuchi, T., Lu, J., Yoshizaki, G. and Satoh, S. 2002. Effect on the growth and body composition of juvenile tilapia Oreochromis niloticus. Fish. Sci., 68(1): 34-40. https://doi. org/10.1046/j.1444-2906.2002.00386.x.

Tartiel, M. B. 2005. Physiological studies on some green algae. $\mathrm{Ph}$. D. thesis, Faculty of Agriculture, Cairo University, Egypt.

Taufek, N. M., Aspani, F., Muin, H., Raji, A. A., Razak, S. A. and Alias, Z. 2016. The effect of dietary cricket meal (Gryllus bimaculatus) on growth performance, antioxidant enzyme activities and haematological response of African catfish (Clarias gariepinus). Fish Physiol. Biochem., 42(4): 1143-1155. https://doi.org/10.1007/s10695-016-0204-8.

Teuling, E., Schrama, J. W., Gruppen, H. and Wierenga, P. A. 2017. Effect of cell wall characteristics on algae nutrient digestibility in Nile tilapia (Oreochromis niloticus) and African catfish (Clarus gariepinus). Aquaculture, 479: 490-500. https://doi.org/10.1016/j aquaculture. 2017.06.025.

Tocher, D. R. 2015. Omega-3 long-chain polyunsaturated fatty acids and aquaculture in perspective. Aquaculture, 449: 94-107. https://doi.org/10.1016/j.aquaculture.2015.01.010.

Tucker, C. S. and Hargreaves, J. A. 2004. Biology and culture of channel catfish, Elsevier. https://doi.org/10.1016/s01679309(04)x8001-7.

Umesh, N., Dathatri, K., Nandeesha, M., Gangadhara, B. and Varghese, T. 1994. Digestibility of dry matter and protein from Spirulina platensis by common carp, Cyprinus carpio, with a note on time of faeces collection in digestibility estimations. Fish nutrition research in Asia, Proceedings of the $5^{\text {th }}$ Asian Fish Nutrition Workshop, Asian Fisheries Society, Manila, Philippines, p. 81-84.

Unprasert, N. G. 1994. Evaluation of the use of "ideal" protein concept to estimate essential amino acid requirements of the Clarias hybrid (Clarias macrocephalus $x$ Clarias gariepinus). Doctoral Dissertation, Mississippi State University, Mississippi, USA, 119 pp.

Uys, W. and Hecht, T. 1985. Evaluation and preparation of an optimal dry feed for the primary nursing of Clarias gariepinus larvae (Pisces: Clariidae). Aquaculture, 47 (2-3): 173-183. https://doi.org/10.1016/0044-8486(85)90063-8.

Vello, V., Phang, S.-M., Chu, W.-L., Majid, N. A., Lim, P.-E. and Loh, S.-K. 2014. Lipid productivity and fatty acid compositionguided selection of Chlorella strains isolated from Malaysia for biodiesel production. J. Appl. Phycol., 26(3): 1399-1413. https://doi.org/10.1007/s10811-013-0160-y. 
Wang, L., Pan, B., Sheng, J., Xu, J. and Hu, Q. 2007. Antioxidant activity of Spirulina platensis extracts by supercritical carbon dioxide extraction. Food Chem., 105: 36-41. https:// doi.org/10.1016/j.foodchem.2007.03.054.

Watanabe, T. 1990. Effect of dietary Spirulina supplement on growth performance and flesh lipids of cultured striped jack. J. Tokyo Univ. Fish., 77: 231-239.

Webster, C. D. and Lim, C. 2002. Nutrient requirements and feeding of finfish for aquaculture, CABI Publishing, Wallingford Oxon, UK, 448 pp.

Xu, W., Gao, Z., Qi, Z., Qiu, M., Peng, J.-Q. and Shao, R. 2014. Effect of dietary Chlorella on the growth performance and physiological parameters of gibel carp, Carassius auratus gibelio. Turk J. Fish. Aquat. Sci., 14(1): 53-57. https://doi. org/10.4194/1303-2712-v14_1_07.
Xue, C., Hu, Y., Saito, H., Zhang, Z., Li, Z., Cai, Y., Ou, C., Lin, H. and Imbs, A. B. 2002. Molecular species composition of glycolipids from Sprirulina platensis. Food Chem., 77(1): 9-13.https://doi.org/10.1016/s0308-8146(01)00315-6.

Yamaguchi, K. 1996. Recent advances in microalgal bioscience in Japan, with special reference to utilisation of biomass and metabolites: a review. J. Appl. Phycol., 8(6): 487-502. https://doi.org/10.1007/bf02186327.

Yildirim-Aksoy, M., Lim, C., Davis, D. A., Shelby, R. and Klesius, P. H. 2007. Influence of dietary lipid sources on the growth performance, immune response and resistance of Nile tilapia, Oreochromis niloticus, to Streptococcus iniae challenge. J. Appl. Aquac., 19(2): 29-49. https://doi. org/10.1300/j028v19n02_02.

Date of Receipt $\quad$ : 22.02.2019

Date of Acceptance : 21.11 .2019 